\title{
Effects of switching from anastrozole to letrozole, due to reimbursement policy, on the outcome of breast cancer therapy
}

This article was published in the following Dove Press journal:

Breast Cancer: Targets and Therapy

27 August 2014

Number of times this article has been viewed

\section{Potchavit Aphinives \\ Damnern Vachirodom \\ Chaiyut Thanapaisal \\ Dhanes Rangsrikajee \\ Ongart Somintara}

Department of Surgery, Faculty of Medicine, Khon Kaen University, Thailand
Correspondence: Potchavit Aphinives Department of Surgery, Faculty of Medicine, Khon Kaen University, 123 Moo 16, Mittraphap Road, Tambon Muang, Khon Kaen 40002,Thailand Email potchavit@gmail.com
Background: Endocrine therapy is one of the standard treatments for estrogen-receptor-positive breast cancer patients. Letrozole is the only aromatase inhibitor (AI) included in Thailand's essential drug list since the change of reimbursement policy in 2008, when patients had to change their AIs (other than letrozole) to letrozole. This study aimed to prove that the efficacy of anastrozole plus letrozole is not less than anastrozole alone.

Methods: All medical records of breast cancer patients taking anastrozole between 2004 and 2013 were reviewed. Some patients were initially treated with anastrozole and then changed to letrozole (A-LTZ group), whereas the other patients were continuously treated with anastrozole until completion of therapy (ANZ group).

Results: In a total of $180(55.9 \%)$ out of the 322 cases, anastrozole was replaced with letrozole. The mean age of patients in the ANZ group was 54.9 years and that of those in the A-LTZ group was 54.2 years. Clinical stages (1-4) of the ANZ versus A-LTZ patients were four versus four, 76 versus 116, 46 versus 46, and 16 versus 14, respectively. ANZ patients took AI monotherapy (46.5\%) and switching strategy (53.5\%), while A-LTZ patients took AI monotherapy $(53.9 \%)$ and switching strategy (46.1\%). The overall survival (OS) of A-LTZ patients was longer than that of ANZ patients. Stage 2 and 4 patients in the A-LTZ group also had better OS than those in the ANZ group, but stage 3 patients had similar OS in both groups.

Conclusion: Anastrozole can be replaced by letrozole any time during endocrine therapy. The patients taking anastrozole plus letrozole surprisingly seemed to have better OS than patients taking anastrozole alone.

Keywords: estrogen-receptor-positive, hormonal-responsive, tamoxifen, reimbursement policy, essential drug list

\section{Introduction}

Endocrine therapy is one of the standard treatments for estrogen-receptor (ER)-positive breast cancer patients. It can be used as an adjuvant for the early stage ${ }^{1}$ or a palliative for the advanced disease. ${ }^{2}$ In Thailand, the available oral anti-estrogen drugs include tamoxifen, anastrozole, letrozole, and exemestane. For premenopausal patients, tamoxifen seems to be the drug of choice, while aromatase inhibitors (AIs) have been used for postmenopausal patients. ${ }^{2-8}$ AIs are divided into two groups: nonsteroidal, which includes anastrozole and letrozole, and steroidal, which includes exemestane. Switching strategy using an AI and tamoxifen is as effective as the AI monotherapy. ${ }^{9,10}$

Since 2002, every Thai person has been covered by one of three medical reimbursement systems: the Civil Servant Medical Benefit Scheme (CSMBS) for government officers, social security (SS) for employees, or universal coverage (UC) for the rest 
of the Thai people. Each system has different guidelines for treatment and overall budgets depending on its rules and policies. However, the essential drug list was established to assure basic standard treatment for all reimbursement systems. Three AIs were initially classified as "essential drugs" in 1999, but only letrozole remained on the essential drug list after 2008. All patients who had taken anastrozole or exemestane under SS and UC were automatically changed to letrozole due to reimbursement policy. Although the benefits of AI therapy appear to be a "class effect", with all drugs having a similar efficacy, ${ }^{11}$ any drug can be selected to treat a patient. However, another group should be used instead of the same group when treatment fails. ${ }^{11}$ There is no evidence of what benefit patients get when they have to change from anastrozole to letrozole without the adverse effects of anastrozole..$^{7-15}$

As such, we studied the effects of switching from anastrozole to letrozole as a result of the reimbursement policy. If both drugs have the same efficacy, patients taking anastrozole plus letrozole should have end results equal to those of patients taking anastrozole alone.

\section{Materials and methods}

All medical records of breast cancer patients taking anastrozole between 2004 and 2013 were reviewed. Some patients were automatically changed from anastrozole to letrozole due to the change of the reimbursement policy. Anastrozole therapy included two types of strategy: monotherapy for 5 years, or 2-3 years of tamoxifen followed by 2-3 years of anastrozole for a total of up to 5 years. Patients were divided into two groups: the ANZ group, which included patients treated with anastrozole (with or without tamoxifen), and the A-LTZ group, which included patients treated with anastrozole (with or without tamoxifen) plus letrozole within 5 years of endocrine therapy. Patients were also categorized by type of reimbursement: CSMBS and non-CSMBS. Demographic data, type of reimbursement, endocrine therapy, and overall survival (OS) were reviewed and analyzed.

Demographic data were analyzed using Excel ${ }^{\circledR} 2007$ (Microsoft, Redmond, WA, USA). Survival data were analyzed using Stata (v 10.1; StataCorp, College Station, TX, USA). OSs were shown by Kaplan-Meier survival curve. Cox regression model was used to compare the OS between the ANZ and A-LTZ groups. A $P$-value $<0.05$ was considered statistically significant.

This study was reviewed and approved by the Khon Kaen University Ethics Committee for Human Research based on the Declaration of Helsinki and the ICH Good Clinical Practice Guidelines.

\section{Results}

The medical records of 322 patients with invasive breast cancer treated with anastrozole were reviewed. There were eight stage 1 patients (mean age $59 \pm 14.1$ years), 192 stage 2 patients (mean age $54.7 \pm 10.4$ years), 92 stage 3 patients (mean age $53.9 \pm 10.5$ years), and 30 stage 4 patients (mean age $54.6 \pm 9.6$ years). In 180 cases $(55.9 \%)$ out of the 322 cases, anastrozole was replaced with letrozole. The mean age of patients in the ANZ group was 54.9 years; in the A-LTZ group, it was 54.2 years. Clinical stages (1-4) of patients in the ANZ versus A-LTZ groups were four versus four, 76 versus 116, 46 versus 46, and 16 versus 14, respectively. The ANZ group took AI monotherapy (46.5\%) and switching strategy (53.5\%), while A-LTZ took AI monotherapy (53.9\%) and switching strategy (46.1\%) (Table 1).

All stage 1 patients survived more than 10 years after diagnosis. The 10 -year survival rate was about $75 \%$ for the stage 2 patients, $50 \%$ for the stage 3 , and $20 \%$ for the stage 4 patients (Table 2 ).

The OS of breast cancer patients in the ANZ and A-LTZ groups was analyzed by Cox regression model, and the OS of the A-LTZ group was found to be significantly better than that of the ANZ group (Figure 1). When the OS was stratified

Table I Comparison of demographic data between the anastrozole (ANZ) group and the anastrozole followed by letrozole (A-LTZ) group

\begin{tabular}{lll}
\hline Characteristic & ANZ (n= I 42) & A-LTZ (n= I 80) \\
\hline $\begin{array}{l}\text { Mean age (years) } \\
\text { Clinical staging }\end{array}$ & $54 \pm$ I0.7 & $54 \pm 10.2$ \\
Stage I & $4(2.86 \%)$ & $4(2.22 \%)$ \\
Stage 2 & $76(53.52 \%)$ & $116(64.44 \%)$ \\
Stage 3 & $46(32.39 \%)$ & $46(25.55 \%)$ \\
Stage 4 & $16(11.26 \%)$ & $14(7.77 \%)$ \\
Type of reimbursement & & \\
CSMBS & $64(45.07 \%)$ & $83(46.11 \%)$ \\
Non-CSMBS & $78(54.93 \%)$ & $97(53.89 \%)$ \\
Endocrine treatment strategy & & \\
Al only & $66(46.48 \%)$ & $97(53.89 \%)$ \\
Stage I & $2(3.03 \%)$ & $2(2.06 \%)$ \\
Stage 2 & $35(53.03 \%)$ & $59(60.82 \%)$ \\
Stage 3 & $19(28.79 \%)$ & $29(29.90 \%)$ \\
Stage 4 & $10(15.15 \%)$ & $7(7.22 \%)$ \\
Al plus tamoxifen & $76(53.52 \%)$ & $83(46.11 \%)$ \\
Stage I & $2(2.63 \%)$ & $2(2.41 \%)$ \\
Stage 2 & $41(53.95 \%)$ & $57(68.67 \%)$ \\
Stage 3 & $27(35.53 \%)$ & $17(20.48 \%)$ \\
Stage 4 & $6(7.89 \%)$ & $7(8.43 \%)$ \\
\hline Ab
\end{tabular}

Abbreviations: Al, aromatase inhibitor; CSMBS, Civil Servant Medical Benefit Scheme. 
Table 2 Overall survival of breast cancer patients

\begin{tabular}{lll}
\hline Stage & 5-year survival $\mathbf{( 9 5 \% ~ C l )}$ & I 0-year survival (95\% CI) \\
\hline $\mathrm{I}$ & $100.00 \%$ & $100.00 \%$ \\
2 & $84.90 \%(79.00-89.25)$ & $75.72 \%(68.69-81.39)$ \\
3 & $67.39 \%(56.79-75.93)$ & $55.37 \%(44.34-65.07)$ \\
4 & $26.67 \%(|2.6|-43.0 I)$ & $19.44 \%(7.62-35.28)$ \\
\hline
\end{tabular}

Abbreviation: $\mathrm{Cl}$, confidence interval.

by each stage, Kaplan-Meier survival curves showed stage 2 and stage 4 patients in the A-LTZ group had a significantly better OS than their stage-matched patients in the ANZ group, although no statistical difference was observed in OS between stage 3 patients in the A-LTZ group and ANZ group (Figures 2-4).

\section{Discussion}

Anastrozole and letrozole are both nonsteroidal AIs. When the breast cancer patient cannot tolerate one of these, we can use another as an alternative. This might be the main reason anastrozole was automatically changed to letrozole when anastrozole was removed from the essential drug list in Thailand. According to previous studies, ${ }^{11,12,15}$ all AIs seem to have similar efficacy. However, this does not mean that we can change any drugs by own favor.

Even after the policy of automatic change from anastrozole to letrozole became effective in 2008, some patients still continued with anastrozole. Theoretically, all patients under SS and UC reimbursement systems had neither the chance to start anastrozole nor to continue anastrozole. But our hospital committee compromised on this: if the remaining period of endocrine therapy was less than 6 months, patients under treatment with anastrozole were allowed to continue their drug until completion of their endocrine therapy, regardless of their type of reimbursement coverage. The patients under the CSMBS were allowed to continue anastrozole or even newly start anastrozole therapy, if their attending physicians considered their patients were better treated with anastrozole rather than letrozole. Without this strategy, the proportion of non-CSMBS patients in the A-LTZ group would have been much higher than in the ANZ group, because the CSMBS covers only $8 \%-10 \%$ of the Thai population. But in our study, the proportion of CSMBS and non-CSMBS patients in the ANZ group (45:56) did not differ from that in the A-LTZ group (46:54).

In addition, some physicians preferred to use the switching strategy rather than AI monotherapy. When the policy changed, some physicians switched anastrozole to tamoxifen for the rest of therapy duration. Consequently, the number of patients treated with letrozole monotherapy has become greater than that with anastrozole monotherapy. Several studies have reported no significant difference in disease-free survival for either switching strategy compared with the AI monotherapy. ${ }^{9,10,16}$ Thus, the different proportion of AI monotherapy and switching strategy between the ANZ and A-LTZ groups may not be the reason for the difference of OS. The recommended switching strategy was to start with tamoxifen for 2-3 years then follow this with an AI. However, several studies have revealed that an AI was not the best therapy for

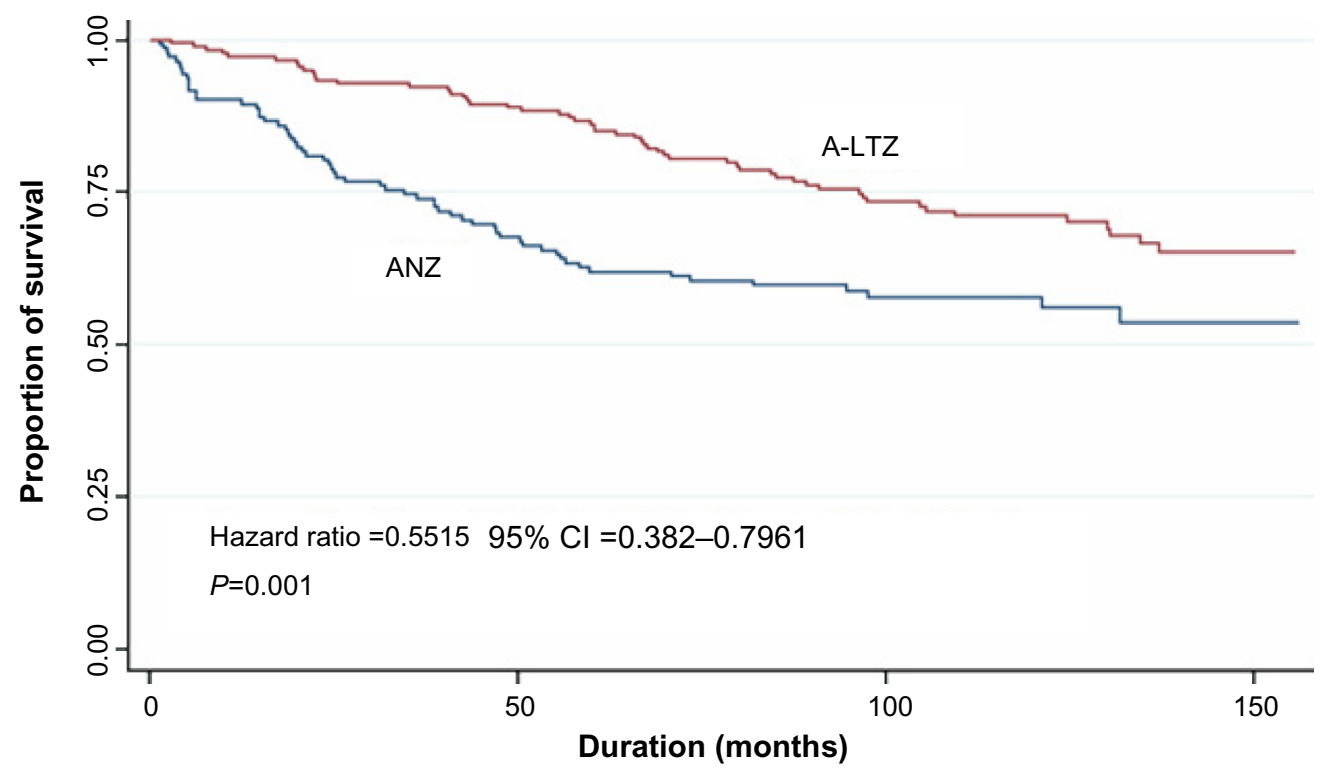

Figure I Kaplan-Meier survival curve of anastrozole-group (ANZ) and the anastrozole followed by letrozole-group (A-LTZ) patients (all stages). Abbreviation: $\mathrm{Cl}$, confidence interval. 


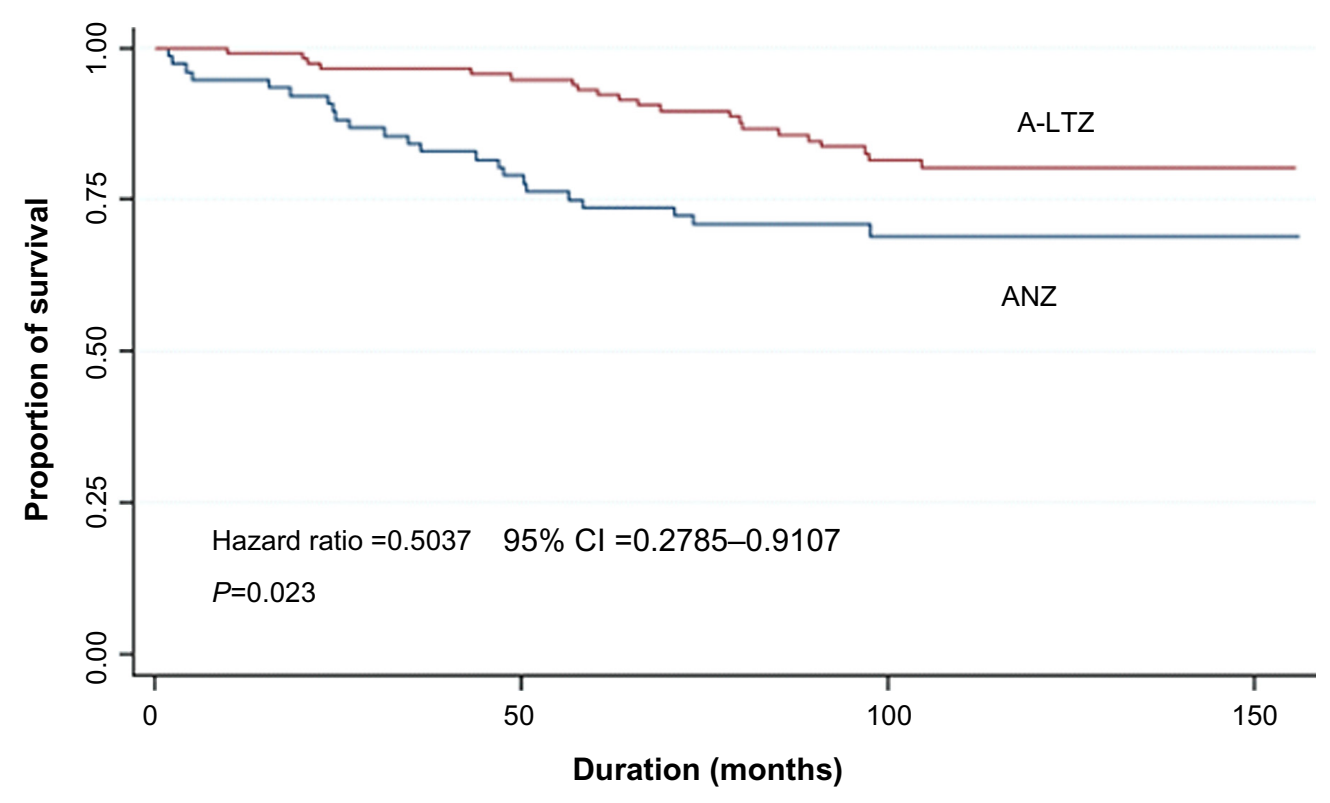

Figure 2 Kaplan-Meier survival curve of stage 2 anastrozole-group (ANZ) and the anastrozole followed by letrozole-group (A-LTZ) patients. Abbreviation: $\mathrm{Cl}$, confidence interval.

all postmenopausal patients with ER-positive early stage breast cancer because AI monotherapy increased the risk of death without recurrence. ${ }^{13,17,18}$ Thus, switching from an AI to tamoxifen was recommended for some patients suffering from AI toxicities.

In the present study, the Kaplan-Meier survival curves showed stage 2 and 4 patients in the ANZ group had a shorter OS than those in the A-LTZ group (Figures 2 and 4), but the OS of stage 3 patients showed no different survival (Figure 3). Why the ANZ group had a shorter OS than the
A-LTZ group remains unknown. An AI is considered to be superior to tamoxifen in terms of disease-free survival, and it might have an OS benefit in breast cancer patients with lymph-node metastasis. ${ }^{8-11}$ The ratio of AI monotherapy to AI plus tamoxifen in the A-LTZ group (54:46) was larger than that in the ANZ group (46:54) (Table 1). This may be responsible for patients in the A-LTZ group having a better outcome than those in the ANZ group. Although we aimed to clarify the non-inferiority of anastrozole plus letrozole to anastrozole alone, we surprisingly realized that patients

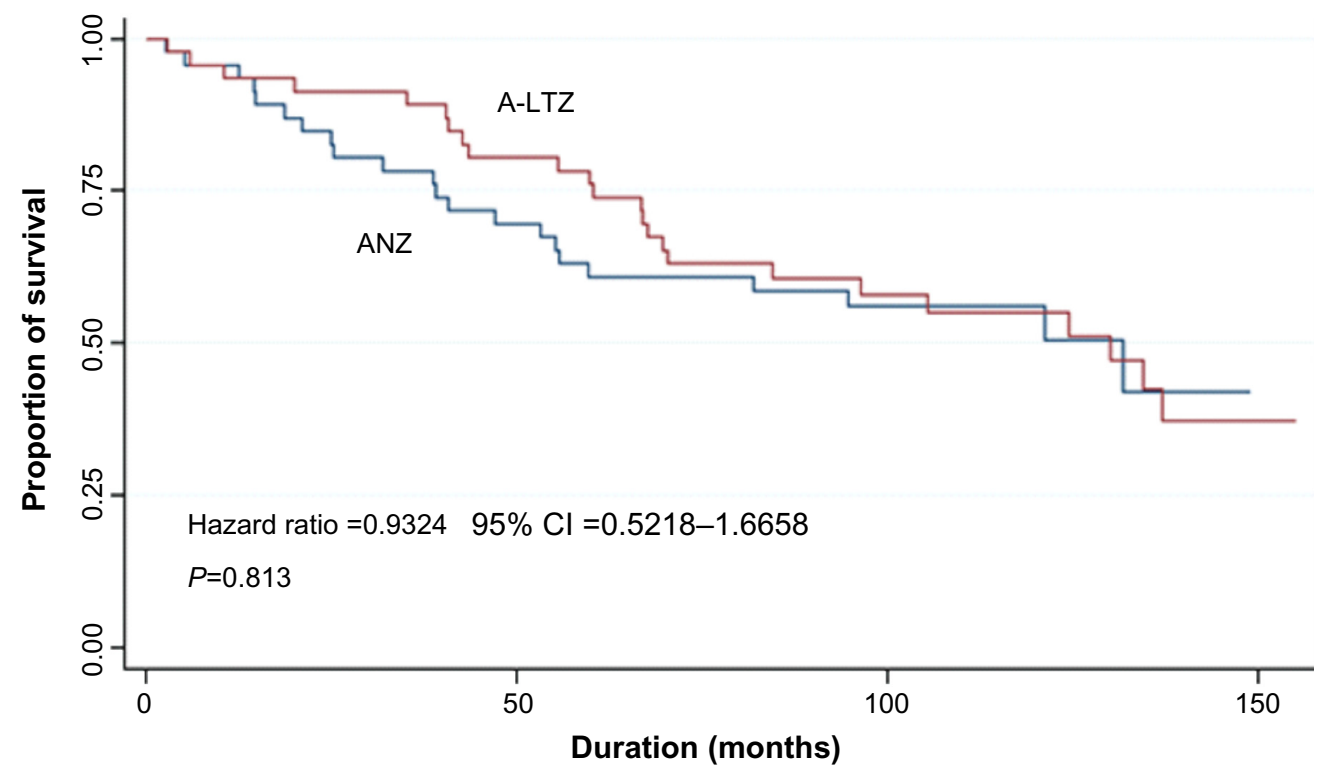

Figure 3 Kaplan-Meier survival curve of stage 3 anastrozole-group (ANZ) and the anastrozole followed by letrozole-group (A-LTZ) patients. Abbreviation: $\mathrm{Cl}$, confidence interval. 


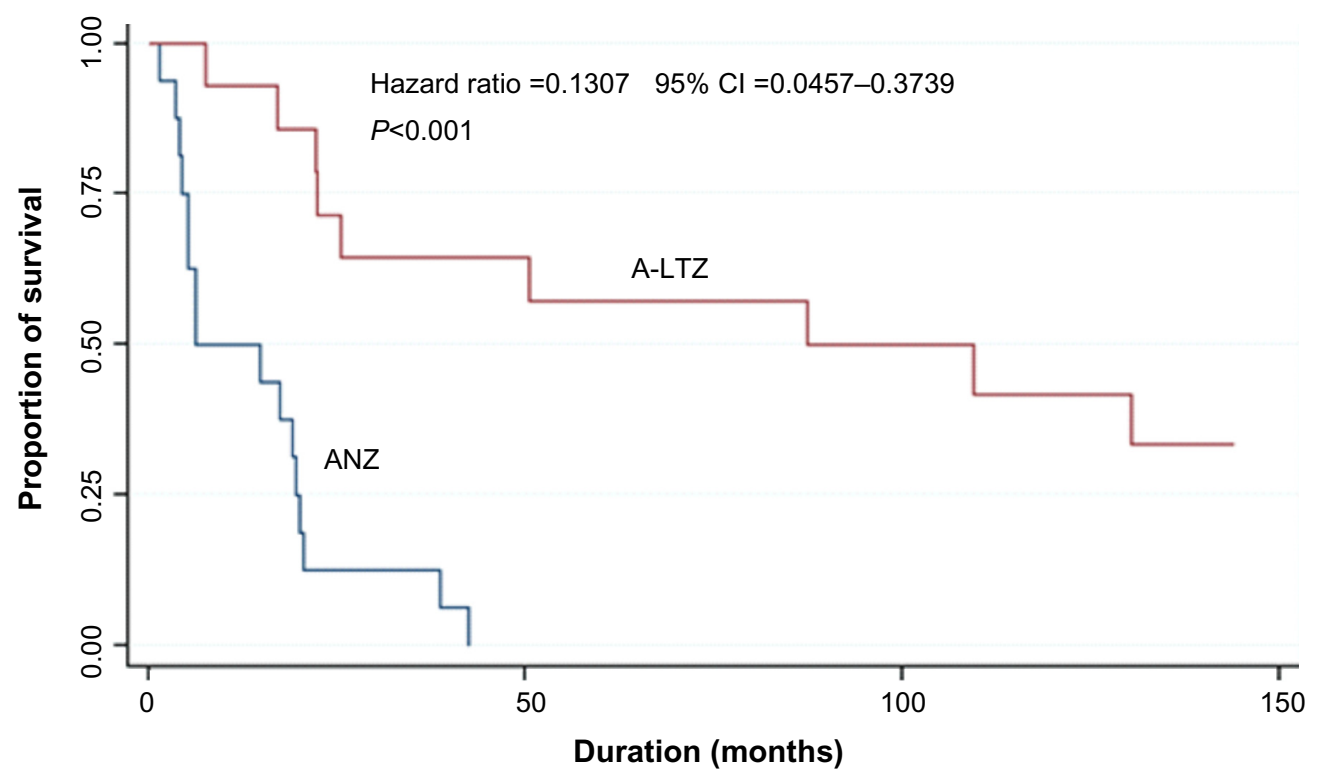

Figure 4 Kaplan-Meier survival curve of stage 4 anastrozole-group (ANZ) and the anastrozole followed by letrozole-group (A-LTZ) patients. Abbreviation: $\mathrm{Cl}$, confidence interval.

taking anastrozole plus letrozole seemed to be better than those taking anastrozole alone. However, while we could not conclude whether anastrozole plus letrozole was superior to or similar to letrozole monotherapy, our study revealed anastrozole plus letrozole was superior to anastrozole monotherapy. Some studies have shown letrozole might be superior to anastrozole in terms of quality of life due to lower incidence of adverse events. ${ }^{19,20}$ Further study with more patients should be performed to confirm the OS difference between treatment with anastrozole alone, letrozole alone, and anastrozole plus letrozole.

\section{Conclusion}

Since 2008, only letrozole has remained in Thailand's essential drug list. The ER-positive invasive breast cancer patients were changed from anastrozole to letrozole due to the change of reimbursement policy. Anastrozole can be replaced by letrozole any time during endocrine therapy. The patients taking anastrozole plus letrozole seemed to have a better OS than those taking anastrozole alone.

\section{Disclosure}

The authors declare no conflicts of interest in this work.

\section{References}

1. Conner C, Attai D. Adjuvant endocrine therapy for the surgeon: options, side effects, and their management. Ann Surg Oncol. 2013;20(10): 3188-3193.

2. Smith IE, Dowsett M. Aromatase inhibitors in breast cancer. New Engl J Med. 2003;348(24):2431-2442.
3. Arimidex, Tamoxifen, Alone or in Combination (ATAC) Trialists' Group, Forbes JF, Cuzick J, et al. Effect of anastrozole and tamoxifen as adjuvant treatment for early-stage breast cancer: 100-month analysis of the ATAC trial. Lancet Oncol. 2008;9(1):45-53.

4. Cuzick J, Sestak I, Baum M, et al; ATAC/LATTE investigators. Effect of anastrozole and tamoxifen as adjuvant treatment for early-stage breast cancer: 10-year analysis of the ATAC trial. Lancet Oncol. 2010;11(12):1135-1141.

5. Davies C, Pan H, Godwin J, et al; Adjuvant Tamoxifen: Longer Against Shorter (ATLAS) Collaborative Group. Long-term effects of continuing adjuvant tamoxifen to 10 years versus stopping at 5 years after diagnosis of oestrogen receptor-positive breast cancer: ATLAS, a randomised trial. Lancet. 2013;381(9869):805-816.

6. Goss PE, Ingle JN, Martino S, et al. A randomized trial of letrozole in postmenopausal women after five years of tamoxifen therapy for early-stage breast cancer. N Engl J Med. 2003;349(19):1793-1802.

7. Jakesz R, Jonat W, Gnant M, et al; ABCSG and the GABG. Switching of postmenopausal women with endocrine-responsive early breast cancer to anastrozole after 2 years' adjuvant tamoxifen: combined results of ABCSG trial 8 and ARNO 95 trial. Lancet. 2005;366(9484): 455-462.

8. Burstein HJ, Prestrud AA, Seidenfeld J, et al; American Society of Clinical Oncology. American Society of Clinical Oncology clinical practice guideline: update on adjuvant endocrine therapy for women with hormone receptor-positive breast cancer. J Clin Oncol. 2010;28(23):3784-3796.

9. van de Velde C, Rea D, Seynaeve C, et al. Adjuvant tamoxifen and exemestane in early breast cancer (TEAM): a randomised phase 3 trial. Lancet. 2011;377(9762):321-331.

10. BIG 1-98 Collaborative Group, Mouridsen H, Giobbie-Hurder A, et al. Letrozole therapy alone or in sequence with tamoxifen in women with breast cancer. N Engl J Med. 2009;361(8):766-776.

11. Fabian CJ. The what, why and how of aromatase inhibitors: hormonal agents for treatment and prevention of breast cancer. Int J Clin Pract. 2007;61(12):2051-2063.

12. Beresford M, Tumur I, Chakrabarti J, Barden J, Rao N, Makris A. A qualitative systematic review of the evidence base for non-crossresistance between steroidal and non-steroidal aromatase inhibitors in metastatic breast cancer. Clin Oncol (R Coll Radiol). 2011;23(3): 209-215. 
13. Fontein DB, Seynaeve C, Hadji P, et al. Specific adverse events predict survival benefit in patients treated with tamoxifen or aromatase inhibitors: an international tamoxifen exemestane adjuvant multinational trial analysis. J Clin Oncol. 2013;31(18):2257-2264.

14. Friese CR, Pini TM, Li Y, et al. Adjuvant endocrine therapy initiation and persistence in a diverse sample of patients with breast cancer. Breast Cancer Res Treat. 2013;138(3):931-939.

15. Berry J. Are all aromatase inhibitors the same? A review of controlled clinical trials in breast cancer. Clin Ther. 2005;27(11):1671-1684.

16. Breast International Group (BIG) 1-98 Collaborative Group, Thürlimann B, Keshaviah A, et al. A comparison of letrozole and tamoxifen in postmenopausal women with early breast cancer. $N \mathrm{Engl}$ J Med. 2005;353(26):2747-2757.
17. Amir E, Seruga B, Niraula S, Carlsson L, Ocana A. Toxicity of adjuvant endocrine therapy in postmenopausal breast cancer patients: a systematic review and meta-analysis. J Natl Cancer Inst. 2011;103(17):1299-1309.

18. Fallowfield L, Cella D, Cuzick J, Francis S, Locker G, Howell A. Quality of life of postmenopausal women in the Arimidex, Tamoxifen, Alone or in Combination (ATAC) Adjuvant Breast Cancer Trial. J Clin Oncol. 2004;22(21):4261-4271.

19. Thomas R. Examining quality of life issues in relation to endocrine therapy for breast cancer. Am J Clin Oncol. 2003;26(4):S40-S44.

20. Thomas R, Godward S, Makris A, Bloomfield D, Moody AM, Williams M. Giving patients a choice improves quality of life: a multicentre, investigator-blind, randomised, crossover study comparing letrozole with anastrozole. Clin Oncol (R Coll Radiol). 2004;16(7):485-491.

\section{Publish your work in this journal}

Breast Cancer: Targets and Therapy is an international, peerreviewed open access journal focusing on breast cancer research, identification of therapeutic targets and the optimal use of preventative and integrated treatment interventions to achieve improved outcomes, enhanced survival and quality of life for the cancer patient.
View the full aims and scopes of this journal here. The manuscript management system is completely online and includes a very quick and fair peer-review system, which is all easy to use. Visit http:// www.dovepress.com/testimonials.php to read real quotes from published authors.

Submit your manuscript here: http://www.dovepress.com/breast-cancer---targets-and-therapy-journal 